\title{
Vine Cuttings Technique for Evaluating the Reaction of Dioscorea rotundata Varieties to Root-Knot Nematodes
}

\author{
Temitayo O. Omotayo ${ }^{1,2}$, Abiodun O. Claudius-Cole ${ }^{1} \&$ Anonio Lopez-Montes ${ }^{2}$ \\ ${ }^{1}$ Department of Crop Protection and Environmental Biology, University of Ibadan, Ibadan, Nigeria \\ ${ }^{2}$ International Institute of Tropical Agriculture, Ibadan, Nigeria \\ Correspondence: Abiodun O. Claudius-Cole, Department of Crop Protection and Environmental Biology, \\ University of Ibadan, Ibadan, Nigeria. E-mail: bi_cole@yahoo.com; b.claudiuscole@gmail.com
}

Received: March 12, $2018 \quad$ Accepted: May 27, $2018 \quad$ Online Published: August 15, 2018

doi:10.5539/jas.v10n9p76 URL: https://doi.org/10.5539/jas.v10n9p76

This study is an output from YIIFSWA Project Phase I, funded by the International Institute of Tropical Agriculture (IITA), Ibadan, Nigeria through a research grant received from Bill and Melinda Gates Foundation. The authors gratefully acknowledge the support from both organizations.

\begin{abstract}
Root-knot nematodes (Meloidogyne spp.) contribute to low productivity and post harvest losses of white yam (Dioscorea rotundata). This study evaluated yam cultivars for resistance to Meloidogyne spp. using plants generated from single node vine cuttings. Forty accessions of $D$. rotundata were selected for the study and laid out in a randomized complete block design with 10 plants per replicate, three replicates and two treatments (Meloidogyne-inoculated and uninoculated). Vines were planted in vertically hanging bags and inoculated four weeks after with 500 eggs of Meloidogyne spp. Plants were harvested sixteen weeks after inoculation and data were collected on weight of tubers, nematode populations in tubers and soil, and nematode damage to tubers. Vine survival was up to $60 \%$, although inoculated vines had lower rates of survival. All the surviving vines produced tubers of various sizes which differed between the control inoculated tubers. Based on galling index (damage) and reproductive factor, five accessions were designated as resistant with the remaining accessions being susceptible. The use of vine-cuttings was found to be effective for screening yam varieties for resistance to Meloidogyne spp.
\end{abstract}

Keywords: Meloidogyne spp., nodal vine cuttings, resistance, yam

\section{Introduction}

The root-knot nematodes (Meloidogyne spp.) are sedentary endo-parasites and are among the most damaging agricultural pests attacking a wide range of crops (Sahebani \& Hadavi, 2008; Hashem \& Abo-Elyousr, 2011). Vegetables, roots and tubers are two of the main affected group of crops on which they cause yield losses mainly in tropical and sub-tropical agriculture (Kiewnick \& Sikora, 2006; Nyczepir \& Thomas, 2009). In West Africa, yam is the most important tuber crop as a main source of income and cash provider for the system, in addition to being a key staple food, particularly in Nigeria, Ghana, Ivory Coast, Benin and Togo (Ile et al., 2006; FAO, 2012). Much of the increase in production is due to increased land cultivation rather than improved productivity. Damage by pests and diseases, especially plant parasitic nematodes and virus diseases are among the major contributors to losses and reduced yield (Odu et al., 2004; Egesei, Onyeka, \& Asiedu, 2007). The edible part of yam is the underground starchy stem called 'tuber' which also serves as the conventional propagules of the crop. Thus, up to $30 \%$ of the previous harvest may be used to plant a new crop. Root-knot infection is one of the main diseases contributing to low yield and postharvest losses of this important crop in Africa (Nwauzor \& Fawole, 1981; Adegbite \& Agbaje, 2007). The underground tubers get infected in the field and the nematode continues to multiply in the periderm and cortex of tubers during storage resulting in tuber deterioration.

Management of the nematode with pesticides is only temporary, as tubers often get infected from field populations of root-knot nematodes. Selection for resistance remains one of the promising and cheaper methods for resource-poor farmers in the region. Screening of yam for resistance to root-knot nematode disease has been reported by Atu, Odurukwe, and Ogbuji (1983), and Coyne and Ross (2014). Using the conventional method of 
growing plants in pots requires the use of pieces of tubers, inoculating with the sufficient quantity of nematode of interest, and evaluating damage in roots and tubers. This conventional method of evaluating yam accessions for resistance requires the use of yam tuber setts (cut tuber pieces) in a standard $5 \mathrm{~kg}$ capacity pot that would require at least 5,000 nematodes using standard protocol. However, there is often a challenge to obtain sufficient tuber as planting material in breeding programmes to sufficiently replicate for effective screening of large populations durin the early stages of the breeding process. Therefore, a method that would use no or few tubers is desirable to achieve the goal of identifying resistance early in the yam breeding programme towards better line selection.

Yam propagation using vine cutting has been reported since the 50s by Correl et al. (1955), and Vander Zaag and Fox (1981) and it was applied and improved by Shiwachi, Kikuno, and Asiedu (2002) and has been used to multiply desired lines to generate enough planting materials in a short time to evaluate yam accessions or varieties in breeding programmes (Otoo et al., 2016). The purpose of this study was to use plants generated through single-node vine cuttings to identify accessions of Dioscorea rotundata with resistance to Meloidogyne spp.

\section{Materials and Methods}

The experiments were conducted at the screenhouse and Nematology laboratory of the International Institute of Tropical Agriculture (IITA). The 40 yam varieties used were obtained from the yam breeding unit of the Institute.

\subsection{Inoculum Source and Preparation}

Meloidogyne spp.was obtained from a field population maintained with Celosia argentea (L.) cultivated in inoculum plots of the Nematology laboratory in IITA. Galled roots of $C$. argentea were washed to remove adhering soil particles and then chopped into 1-2 cm pieces. The eggs/juveniles of Meloidogyne spp. were extracted from the chopped root pieces using $0.5 \%$ sodium hypochlorite according to the method of Hussey and Barker (1973). The number of eggs/juveniles was counted with the aid of a counting dish while viewing under compound microscope using $10 \times$ magnification. The nematode suspension was adjusted to 500 eggs/juveniles per $\mathrm{ml}$ in a beaker required for the inoculation procedure.

\subsection{Vine Cutting Preparation}

Vines were obtained from the mother plants growing in pots in the screenhouse which were planted for the purpose of generating vines. Vines were collected when plants at 90 and 100 days after sprouting from the setts, when the main stems had grown lateral branches. The length of each vine cutting below the node was $1 \mathrm{~cm}$ and above the node was $2 \mathrm{~cm}$ (Plate 1). Vines were washed and treated with a solution of Mancozeb 80\% and Chloropyrofos $48 \%$ in a container with 10 liters of water to eliminate fungi, mites and insects.

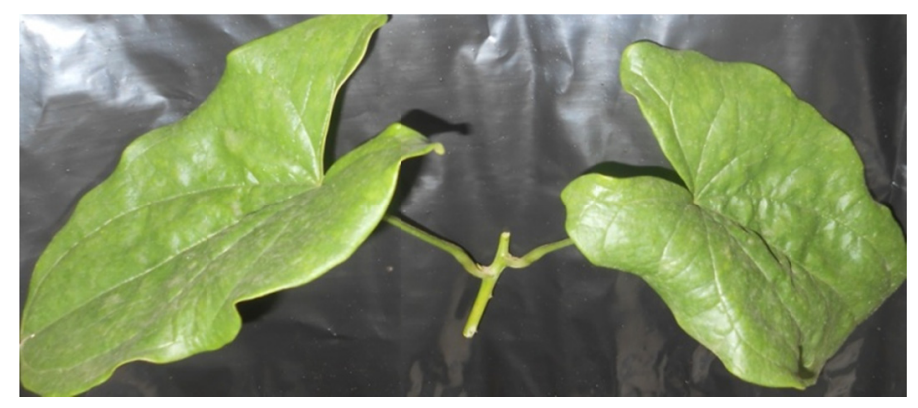

Plate 1 . Vine cutting of yam with a single node

\subsection{Vertical Sacks (Bag) Preparation}

The media for planting was a mixture top soil and river sand in the ratio $2: 1$. The mixture was steam- sterilized at 80- $95{ }^{\circ} \mathrm{C}$ for 2 hours. The sterilized soil was left to cool and stabilize for 10 days after which they were filled into the black polyethylene bags. The bags were $80 \mathrm{~cm} \times 30 \mathrm{~cm}$ with the capacity of containing about 20,000 $\mathrm{cm}^{3}$ of soil but was filled with $15,0000 \mathrm{~cm}^{3}$ of the soil-sand mixture. The opened end of each sack was tied and bound with strong long strings. The long strings were used to hang the bags from the metal beam of the 
screenhouse. One set of bags were hung $2 \mathrm{~m}$ above the floor and another were $50 \mathrm{~cm}$ above the floor thus creating 2 planes of hanging sacks and conserving space.

\subsection{Planting and Inoculation of Vines in the Screenhouse}

Planting was carried out by inserting one vine each into the 10 planting holes made on the surface of the vertical hanging bags. A glass rod of 0.5 diameter was used to make a $1 \mathrm{~cm}$ deep planting hole in the media in which vines were planted. Four weeks after planting, inoculation was carried out by pipetting 500 eggs of Meloidogyne spp. per vine into the soil in which vines were growing thus; making a total of 5000 eggs of Meloidogyne spp. per bag. Uninoculated plants served as the control. Ten vines were planted in separate holes per bag in a randomized complete block design with three replicates (three bags per cultivar). Treatments were 40 yam varieties and each bag contained a single variety. Bags with inoculated plants were separated from uninoculated (control) bags by a row of bags with no plants. The experiment was conducted a second time following the same procedure.

\subsection{Data Collection and Analysis}

The number of surviving vines was counted at four weeks after inoculation and the percentage survival calculated. The number of leaves per plant was also counted. Six months after planting, the plants were harvested, and tubers were weighed and scored for symptoms of root-knot nematode damage using a scale of 1 to 5 , where, $1=0 \%, 2=1-10 \%, 3=11-30 \%, 4=31-60 \%, 5=61-100 \%$ (Claudius-Cole, 2005). The roots and tubers were separated from soil, washed, drained over paper towels and weighed. The roots were cut into $1 \mathrm{~cm}$ pieces for nematode extraction. Extraction from roots was undertaken following maceration in $0.5 \%$ sodium hypochlorite for 5 seconds in a Warring ${ }^{\circledR}$ laboratory blender. The suspension was shaken for three minutes and passed through nested sieves of $2 \mathrm{~mm}$ sieve to remove debris, then through a $60 \mu \mathrm{m}$ sieve for female nematodes, and $28 \mu \mathrm{m}$ sieve for second stage juveniles and eggs. The contents of each sieve were rinsed out with a wash bottle and collected in labeled sample cups. Yam tubers were washed, weighed and peeled. The peels were weighed, chopped into $1-2 \mathrm{~cm}$ and mixed. Nematodes were extracted from the chopped peels as above. Nematode counts were used to determine populations in roots and tubers. The soil of $100 \mathrm{~cm}^{3}$ from the rhizosphere of each vine was collected and bulked per bag. The bulked soil was thoroughly mixed and a $100 \mathrm{~cm}^{3}$ sample was taken out for extraction using the modified Baermann tray method (Coyne et al., 2007). Extracted nematodes from both plant and soil were counted from the resulting extract. The total number of nematodes in soil was estimated for the $1 \mathrm{~kg}$ bulked soil and summed with the number of nematodes counted from plant root and tubers. Reproductive factor was thereafter calculated using

$\mathrm{RF}=\mathrm{Pf} / \mathrm{Pi}$ where $\mathrm{Pf}$ is the total final nematode population per variety and $\mathrm{Pi}$ is the initial inoculum. Host status was assigned based on a modified scheme following the Canto-Saenz (1983) scheme, Resistant $=R F \leq 1, \mathrm{GI} \leq 2$; Tolerant $=\mathrm{RF} \leq 1, \mathrm{GI} \geq 2$; Susceptible $=\mathrm{RF} \geq 1, \mathrm{GI} \geq 2$.

Data on nematode counts were transformed using $\sqrt{\mathrm{x}+1}$ before analysis. Data collected were submitted for analysis of variance for all the treatments and the means were separated using the Student-Newman-Keuls Test at $\mathrm{P}=.05$. Statistical analysis was conducted using SAS program (SAS Institute Inc., 2014).

\section{Results}

The number of leaves were not significantly different between inoculated and uninoculated plants $(\mathrm{P}=0.86)$. In the first trial, percentage survival of plants from bags with Meloidogyne-inoculated plants was lower $(\mathrm{P}=0.05)$ compared to uninoculated plants, and in the second trial, the difference was not significant (Figure 1). The number of tubers was more in the second compared to the first trial but the differences between treatments were not significant. The tuber weight in the second trial was also greater than in the first, however tubers from uninoculated plants weighted more than those produced from inoculated plants. The difference was however significant only in the second trial.

Tuber weight of the yam cultivars varied in both trials. Differences in tuber weight between inoculated and uniniculated plants also varied widely in the two trials (Table 1). In addition two cultivars used in the first trial were unavailable in the second trial, while four cultivars included in the second trial were unavailable during the period of the first trail. Yam variety TDr 99/02562, TDr 02/00515, and TDr 06-15 had significantly $(\mathrm{P}=0.05)$ more tuber weight among inoculated accessions in the first trial (Table 1) than other accessions although they were not significantly higher than Ufenyi and TDr 03/00058. Uninoculated (control) plants in the first trial for cultivars TDr 99/02562, TDr 89/02674, TDr 03/0019, TDr 89/02157 and TDr 97/00917 had significantly (P = 0.05) more yield than other accessions except for TDr 89/02665 and TDr 97/00793. In the second trial, 
inoculated Alumaco and TDr 97/00917 had significantly $(\mathrm{P}=0.05)$ heavier tuber weight compared to most (24) of the accessions.
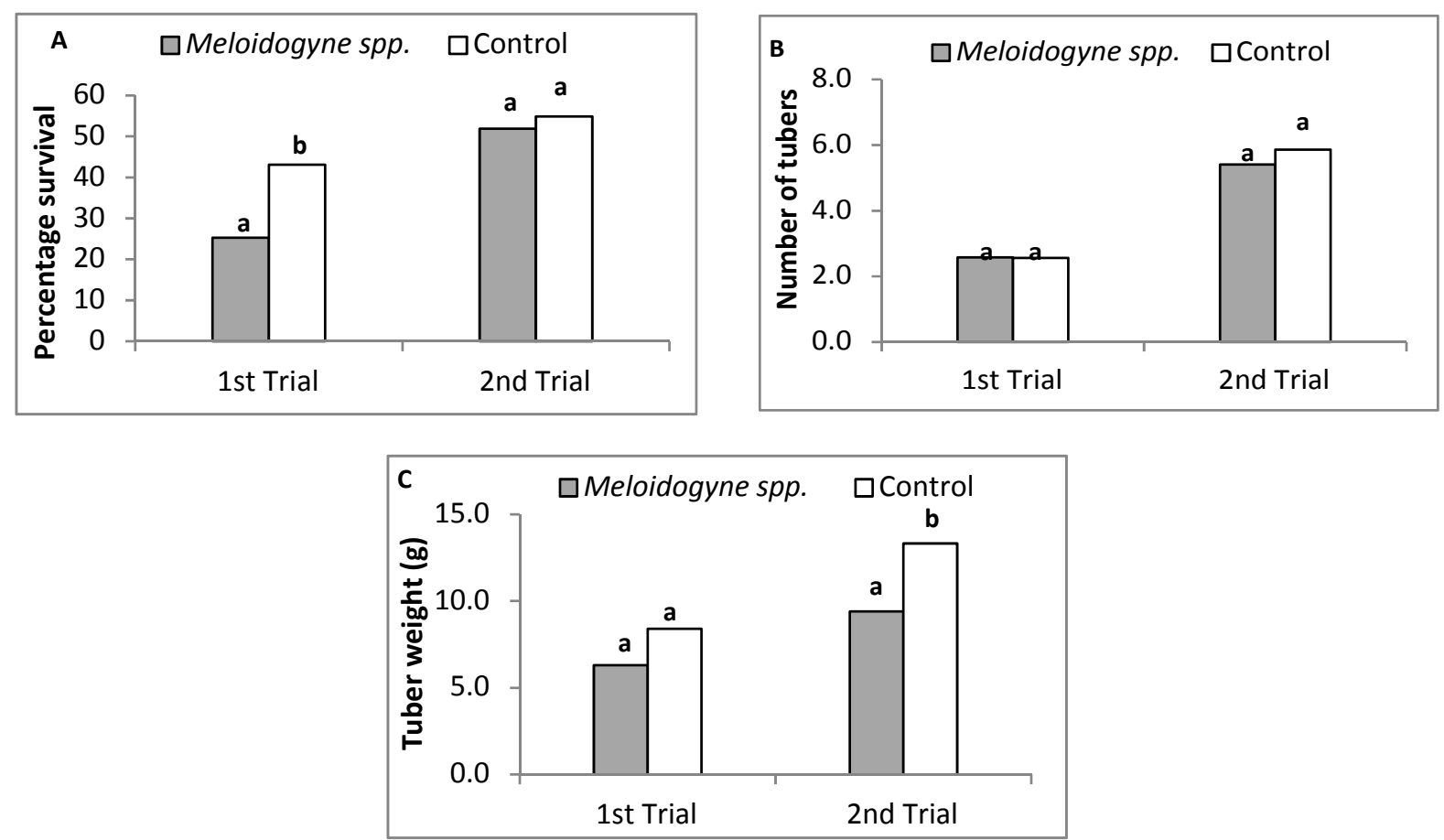

Figure 1. Percentage vine survival (A), and number (B) and weight of tubers (C) of yam plants inculated with Meloidogyne spp. using single node vine cuttings in two trials

For uninoculated (control) plants in the second trial, the yield of TDr97/00917 was significantly $(P=0.05)$ higher than eight other accessions but not significantly different from the remaining accessions. There were no significant differences between tuber weight of inoculated versus control in 13 and 12 of the varieties in the first and second trials respectively. Of these, only two (TDr 96/00582 and TDr 99/02789) reacted similarly in both trials. Where significant differences occurred, uninoculated tubers generally weighed more than inoculated tubers although greater tuber weight was observed in 10 of the cultivars that were inoculated compared to their control in the first trial while 12 cultivars fell into this category in the second trial.

Galling index, nematode populations and nematode reproductive factor were significantly higher in tubers from inoculated bags compared to the control (Figure 2). Trends for galling index and reproductive factor were similar in both trials, however the number of nematode recovered from roots and tubers in the second trial was greater than the first trial.

The tubers harvested from the first trial were not heavily galled $(\mathrm{GI}=1-2)$ the most heavily galled tubers were from TDr 89/02677 with GI of 2.75 (Table 2). Nematode populations were low, and reproductive factor was $\leq 1$ for cultivars TDr 98/00205, TDr 07/00168, TDr 03/00180, TDr 08-3-6, and TDr 01/00405 and were designated as resistant in the first trial (Table 1). Cultivars TDr 94/01108 and TDr 99/02562 were designated as tolerant due to reproductive factor $>1$. Among the susceptible cultivars, TDr $89 / 02672$ had the highest nematode populations and reproductive factor. Similar to the first trial, TDr 98/00205, TDr 03/00180. 
Table 1. Tuber weight (g) of yam varieties inoculated with Meloidogyne spp. in the first and second trial

\begin{tabular}{|c|c|c|c|c|c|c|}
\hline \multirow{3}{*}{$\begin{array}{l}\text { Accession } \\
\text { TDr 00/00539 }\end{array}$} & \multicolumn{3}{|c|}{ First Trial } & \multicolumn{3}{|c|}{ Second Trial } \\
\hline & \multirow{2}{*}{$\begin{array}{l}\text { Control } \\
-\end{array}$} & \multicolumn{2}{|c|}{$\begin{array}{l}\text { Meloidogyne } \\
\text {-inoculated }\end{array}$} & \multirow{2}{*}{$\begin{array}{l}\text { Control } \\
1.05\end{array}$} & \multicolumn{2}{|c|}{$\begin{array}{l}\text { Meloidogyne } \\
\text {-inoculated }\end{array}$} \\
\hline & & - & - & & 1.38 & $*$ \\
\hline TDr 96/00604 & - & - & - & 1.55 & 0.86 & $*$ \\
\hline TDr 97/00925 & - & - & - & 1.72 & 1.92 & ns \\
\hline TDr 97/01715 & - & - & - & 0.93 & 0.44 & ns \\
\hline TDr 96/00582 & $0.11^{\mathrm{c}}$ & $0.07^{\mathrm{b}}$ & ns & $0.66^{\mathrm{b}}$ & $0.90^{\mathrm{c}}$ & $\mathrm{ns}$ \\
\hline Makakusa & $0.14 \mathrm{c}$ & $1.12 \mathrm{~b}$ & $*$ & $2.19^{\mathrm{ab}}$ & $0.96^{\mathrm{bc}}$ & $*$ \\
\hline Alumaco & $2.15^{\mathrm{ab}}$ & $1.82^{\mathrm{b}}$ & $*$ & $2.22^{\mathrm{b}}$ & $4.17^{\mathrm{a}}$ & $*$ \\
\hline TDr 95/19158 & $0.15^{\mathrm{c}}$ & $0.72^{\mathrm{b}}$ & $*$ & $2.70^{\mathrm{ab}}$ & $1.64^{b c}$ & $*$ \\
\hline TDr 99/02789 & $0.15^{\mathrm{c}}$ & $0.11^{\mathrm{b}}$ & ns & $1.27^{\mathrm{ab}}$ & $1.41^{\mathrm{bc}}$ & $\mathrm{ns}$ \\
\hline TDr 89/02677 & $0.16^{\mathrm{c}}$ & $1.42^{\mathrm{b}}$ & $*$ & $0.79^{\mathrm{b}}$ & $1.68^{b c}$ & $*$ \\
\hline TDr 98/00933 & $0.17^{\mathrm{c}}$ & $0.93^{\mathrm{b}}$ & ns & $2.73^{\mathrm{ab}}$ & $1.30^{\mathrm{bc}}$ & $*$ \\
\hline TDr 08-3-6 & $0.18^{\mathrm{c}}$ & $0.02^{\mathrm{b}}$ & ns & $2.50^{\mathrm{ab}}$ & $1.54^{\mathrm{bc}}$ & $*$ \\
\hline Agbawonbe & $0.19 \mathrm{c}$ & $0.78 b$ & ns & - & - & - \\
\hline TDr 99/02607 & $0.19^{c}$ & $0.82^{\mathrm{b}}$ & ns & $0.77^{\mathrm{b}}$ & $1.32^{\mathrm{bc}}$ & $*$ \\
\hline TDr 89/02475 & $0.20^{\mathrm{c}}$ & $1.17^{\mathrm{b}}$ & $*$ & $1.42^{\mathrm{ab}}$ & $0.75^{\mathrm{c}}$ & $*$ \\
\hline TDr 95/18544 & $0.24^{\mathrm{c}}$ & $0.08^{\mathrm{b}}$ & ns & $1.39^{\mathrm{ab}}$ & $0.89^{\mathrm{c}}$ & $*$ \\
\hline TDr 95/01932 & $0.25^{\mathrm{c}}$ & $0.07^{\mathrm{b}}$ & ns & $2.14^{\mathrm{ab}}$ & $1.52^{\mathrm{bc}}$ & $*$ \\
\hline TDr 01/00405 & $0.28^{\mathrm{c}}$ & $1.43^{\mathrm{b}}$ & $*$ & $1.84^{\mathrm{ab}}$ & $1.55^{\mathrm{bc}}$ & $\mathrm{ns}$ \\
\hline TDr 98/00205 & $0.28^{\mathrm{c}}$ & $0.20^{\mathrm{b}}$ & $\mathrm{ns}$ & $1.12^{\mathrm{ab}}$ & $0.37^{\mathrm{c}}$ & $*$ \\
\hline Pouna & $0.32 \mathrm{c}$ & $0.96^{\mathrm{b}}$ & $*$ & - & - & - \\
\hline TDr 95/19177 & $0.32^{\mathrm{c}}$ & $1.31^{\mathrm{b}}$ & * & $1.47^{\mathrm{ab}}$ & $1.22^{\mathrm{bc}}$ & ns \\
\hline TDr 02/00515 & $0.40^{\mathrm{c}}$ & $7.22^{\mathrm{a}}$ & $*$ & $1.80^{\mathrm{ab}}$ & $1.59^{\mathrm{bc}}$ & ns \\
\hline TDr 07/00168 & $0.41^{\mathrm{c}}$ & $0.06^{\mathrm{b}}$ & $\mathrm{ns}$ & $2.02^{\mathrm{ab}}$ & $1.22^{\mathrm{bc}}$ & $*$ \\
\hline Amula & $0.43^{\mathrm{c}}$ & $0.85^{\mathrm{b}}$ & $\mathrm{ns}$ & $2.22^{\mathrm{ab}}$ & $4.17^{\mathrm{a}}$ & * \\
\hline TDr 96/01817 & $0.49^{\mathrm{c}}$ & $0.07^{\mathrm{b}}$ & $*$ & $0.88^{\mathrm{b}}$ & $1.87^{\mathrm{bc}}$ & * \\
\hline TDr 94/01108 & $0.52^{\mathrm{bc}}$ & $0.02^{\mathrm{b}}$ & * & $1.38^{\mathrm{ab}}$ & $1.75^{\mathrm{bc}}$ & * \\
\hline TDr 99/02562 & $0.80^{\mathrm{bc}}$ & $7.80^{\mathrm{a}}$ & $*$ & $2.14^{\mathrm{ab}}$ & $1.59^{\mathrm{bc}}$ & * \\
\hline TDr 03/00180 & $0.90^{\mathrm{bc}}$ & $0.08^{\mathrm{b}}$ & * & $1.02^{\mathrm{ab}}$ & $0.66^{\mathrm{c}}$ & $*$ \\
\hline TDr 03/00058 & $0.91^{\mathrm{bc}}$ & $3.45^{\mathrm{ab}}$ & $*$ & $0.95^{\mathrm{b}}$ & $0.63^{\mathrm{c}}$ & ns \\
\hline TDr 97/00940 & $0.97^{\mathrm{bc}}$ & $1.30^{\mathrm{b}}$ & $*$ & $1.05^{\mathrm{ab}}$ & $2.10^{\mathrm{b}}$ & $*$ \\
\hline TDr 97/00840 & $1.21^{\mathrm{bc}}$ & $0.96^{\mathrm{b}}$ & $*$ & $1.47^{\mathrm{ab}}$ & $2.24^{\mathrm{b}}$ & $*$ \\
\hline TDR 06-4 & $1.42^{\mathrm{b}}$ & $1.86^{\mathrm{b}}$ & ns & - & - & - \\
\hline Ufenyi & $1.48^{\mathrm{b}}$ & $4.51^{\mathrm{ab}}$ & $*$ & $1.21^{\mathrm{ab}}$ & $1.12^{\mathrm{bc}}$ & ns \\
\hline TDr 07/00873 & $2.13^{\mathrm{ab}}$ & $0.10^{\mathrm{b}}$ & $*$ & $1.60^{\mathrm{ab}}$ & $2.25^{\mathrm{b}}$ & $*$ \\
\hline TDr 00/00362 & $2.17^{\mathrm{ab}}$ & $0.69^{\mathrm{b}}$ & $*$ & $1.21^{\mathrm{ab}}$ & $1.37^{\mathrm{b}}$ & ns \\
\hline TDr 89/02672 & $2.38^{\mathrm{ab}}$ & $1.10^{\mathrm{b}}$ & $*$ & $1.88^{\mathrm{ab}}$ & $1.74^{\mathrm{bc}}$ & $\mathrm{ns}$ \\
\hline TDr $89 / 02665$ & $2.50^{\mathrm{ab}}$ & $1.55^{\mathrm{b}}$ & $*$ & $1.92^{\mathrm{ab}}$ & $2.39^{\mathrm{b}}$ & $*$ \\
\hline TDr 06-15 & $2.58^{\mathrm{ab}}$ & $6.23^{\mathrm{a}}$ & $*$ & $1.71^{\mathrm{ab}}$ & $0.37^{\mathrm{c}}$ & * \\
\hline TDr $00 / 00403$ & $2.84^{\mathrm{ab}}$ & $0.21^{\mathrm{b}}$ & * & $0.75^{\mathrm{b}}$ & $2.82^{\mathrm{b}}$ & $*$ \\
\hline TDr 97/00793 & $2.94^{\mathrm{ab}}$ & $0.23^{\mathrm{b}}$ & $*$ & $1.38^{\mathrm{ab}}$ & $0.96^{\mathrm{c}}$ & $*$ \\
\hline TDr 99/02674 & $3.84^{\mathrm{a}}$ & $1.72^{\mathrm{b}}$ & * & $1.42^{\mathrm{ab}}$ & $1.09^{\mathrm{bc}}$ & $*$ \\
\hline TDr 03/00196 & $4.01^{\mathrm{a}}$ & $1.70^{\mathrm{b}}$ & * & $2.40^{\mathrm{ab}}$ & $1.14^{\mathrm{bc}}$ & $*$ \\
\hline TDr 89/02157 & $7.33^{\mathrm{a}}$ & $1.16^{\mathrm{b}}$ & $*$ & $1.07^{\mathrm{ab}}$ & $0.84^{\mathrm{c}}$ & $*$ \\
\hline TDr 97/00917 & $7.91^{\mathrm{a}}$ & $1.07^{\mathrm{b}}$ & $*$ & $3.81^{\mathrm{a}}$ & $1.19^{\mathrm{bc}}$ & $*$ \\
\hline
\end{tabular}

Note. Means with same letter in a row are not significantly different at $\mathrm{P}=0.05$ using the Student-Newman-Keuls Test; $\mathrm{ns}=$ no significant difference, ${ }^{*}=$ significant difference between treatments; $\mathrm{TDr}=$ Tropical Dioscorea rotundata. 

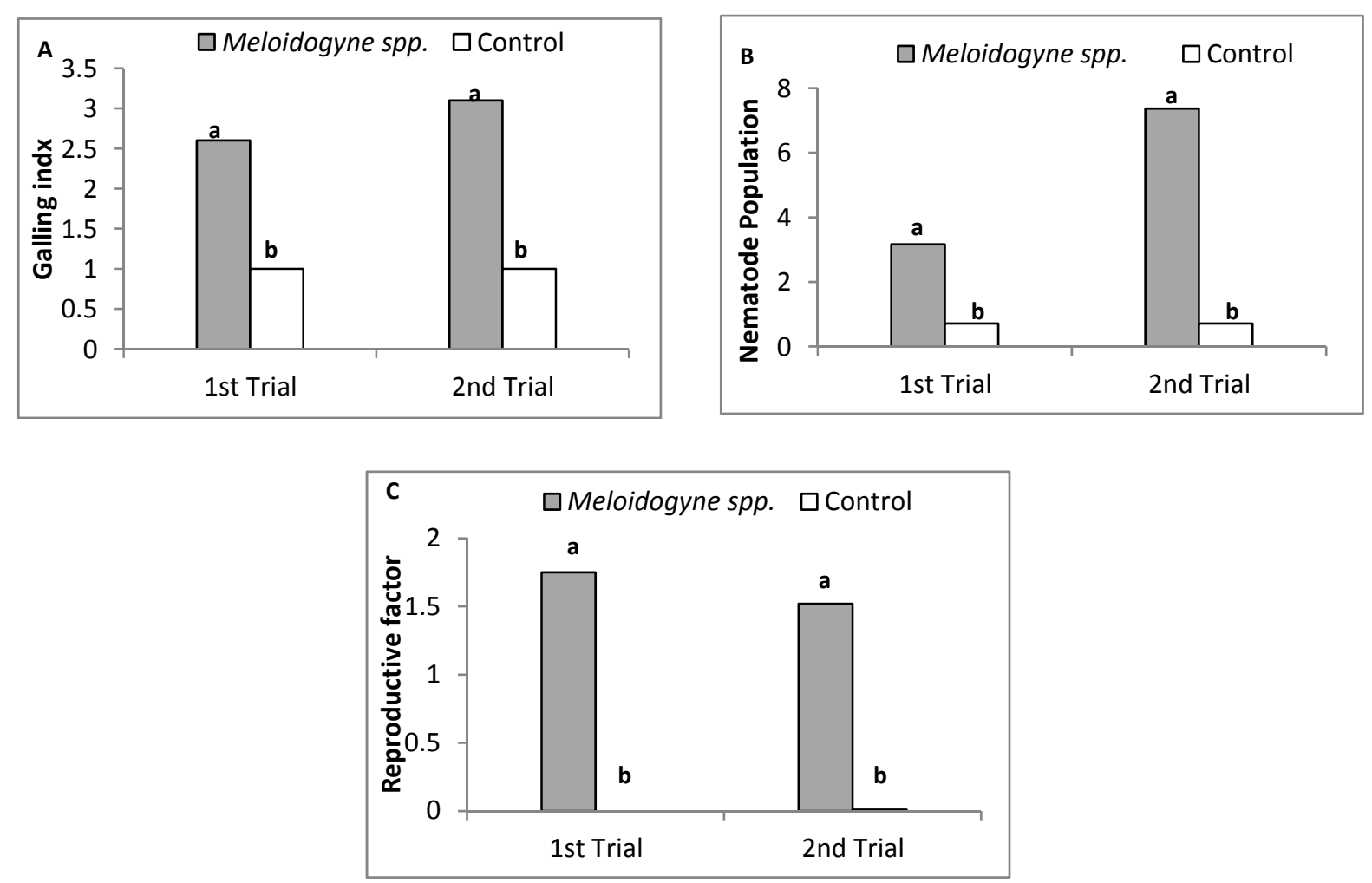

Figure 2. Damage and nematode populations of tubers infected with Meloidogyne spp. in two trails of the nodal vine cutting system 
Table 2. Nematode population, gall index, reproductive factor and host status of accessions innoculated with Meloidogyne spp. in the first Trial

\begin{tabular}{|c|c|c|c|c|}
\hline Accession & Gall-Index & Nematode Count & Reproductive factor & Host status \\
\hline TDr 98/00205 & $1.00^{\mathrm{c}}$ & $3533.19^{d}$ & $0.71^{\mathrm{c}}$ & Resistant \\
\hline TDr $07 / 00168$ & $1.00^{\mathrm{c}}$ & $3268.14^{\mathrm{d}}$ & $0.65^{\mathrm{c}}$ & Resistant \\
\hline TDr $03 / 00180$ & $1.50^{\mathrm{bc}}$ & $4769.11^{\mathrm{d}}$ & $0.95^{\mathrm{c}}$ & Resistant \\
\hline TDr 08-3-6 & $1.00^{\mathrm{c}}$ & $3709.06^{\mathrm{d}}$ & $0.74^{\mathrm{c}}$ & Resistant \\
\hline TDr $01 / 00405$ & $1.25^{\mathrm{bc}}$ & $4125.28^{\mathrm{cd}}$ & $0.83^{b c}$ & Resistant \\
\hline TDr 94/01108 & $1.50^{\mathrm{bc}}$ & $4834.25^{\mathrm{d}}$ & $1.01^{\mathrm{c}}$ & Resistant \\
\hline TDr 99/02562 & $1.50^{\mathrm{bc}}$ & $5299.37^{\mathrm{d}}$ & $1.06^{\mathrm{c}}$ & Tolerant \\
\hline TDr $07 / 00873$ & $2.00^{\mathrm{ab}}$ & $6623.11^{\mathrm{d}}$ & $1.33^{\mathrm{bc}}$ & Susceptible \\
\hline TDr 95/01932 & $2.00^{\mathrm{c}}$ & $7948.10^{\mathrm{cd}}$ & $1.59^{\mathrm{bc}}$ & Susceptible \\
\hline TDr 95/19177 & $2.00^{\mathrm{ab}}$ & $8478.01^{\mathrm{cd}}$ & $1.70^{\mathrm{bc}}$ & Susceptible \\
\hline Makakusa & $2.00^{\mathrm{ab}}$ & $8655.23^{\mathrm{cd}}$ & $1.73^{\mathrm{bc}}$ & Susceptible \\
\hline TDr 95/19158 & $1.25^{\mathrm{bc}}$ & $8743.41^{\mathrm{cd}}$ & $1.75^{\mathrm{bc}}$ & Susceptible \\
\hline TDr 06-4 & $2.00^{\mathrm{ab}}$ & $9979.57^{\mathrm{cd}}$ & $2.00^{\mathrm{b}}$ & Susceptible \\
\hline Alumaco & $1.25^{\mathrm{bc}}$ & $12540.08^{\mathrm{cd}}$ & $2.51^{\mathrm{b}}$ & Susceptible \\
\hline TDr 00/00362 & $2.00^{\mathrm{ab}}$ & $12717.06^{\mathrm{cd}}$ & $2.54^{\mathrm{b}}$ & Susceptible \\
\hline TDr 97/00840 & $2.00^{\mathrm{ab}}$ & $12894.25^{\mathrm{cd}}$ & $2.58^{\mathrm{b}}$ & Susceptible \\
\hline TDr $89 / 02677$ & $2.75^{\mathrm{a}}$ & $13865.18^{\mathrm{cd}}$ & $2.77^{\mathrm{b}}$ & Susceptible \\
\hline TDr $02 / 00515$ & $2.00^{\mathrm{ab}}$ & $14130.42^{\text {cd }}$ & $2.83^{\mathrm{b}}$ & Susceptible \\
\hline TDr 96/00582 & $2.00^{\mathrm{ab}}$ & $15366.27^{\mathrm{cd}}$ & $3.07^{\mathrm{b}}$ & Susceptible \\
\hline TDr 99/02789 & $2.00^{\mathrm{ab}}$ & $15543.05^{\mathrm{cd}}$ & $3.11^{\mathrm{ab}}$ & Susceptible \\
\hline TDr 03/00196 & $2.00^{\mathrm{ab}}$ & $15720.41^{\mathrm{cd}}$ & $3.14^{\mathrm{ab}}$ & Susceptible \\
\hline TDr $89 / 02157$ & $2.00^{\mathrm{ab}}$ & $16161.12^{b c}$ & $3.23^{\mathrm{ab}}$ & Susceptible \\
\hline Agbawonbe & $2.00^{\mathrm{ab}}$ & $16691.07^{\mathrm{bc}}$ & $3.34^{\mathrm{ab}}$ & Susceptible \\
\hline TDr 89/02475 & $2.00^{\mathrm{ab}}$ & $17044.04^{\mathrm{bc}}$ & $3.41^{\mathrm{ab}}$ & Susceptible \\
\hline TDr 99/02607 & $2.00^{\mathrm{ab}}$ & $17574.15^{\mathrm{bc}}$ & $3.51^{\mathrm{ab}}$ & Susceptible \\
\hline Ufenyi & $1.25^{\mathrm{bc}}$ & $17574.36^{\mathrm{bc}}$ & $3.51^{\mathrm{ab}}$ & Susceptible \\
\hline TDr 98/00933 & $1.25^{\mathrm{bc}}$ & $18016.43^{b c}$ & $3.60^{\mathrm{ab}}$ & Susceptible \\
\hline TDr 97/00917 & $2.00^{\mathrm{ab}}$ & $18634.06^{\mathrm{bc}}$ & $3.73^{\mathrm{ab}}$ & Susceptible \\
\hline Amula & $2.00^{\mathrm{ab}}$ & $18899.08^{\mathrm{bc}}$ & $3.78^{\mathrm{ab}}$ & Susceptible \\
\hline TDr $03 / 00058$ & $2.00^{\mathrm{ab}}$ & $19164.23^{\mathrm{bc}}$ & $3.83^{\mathrm{ab}}$ & Susceptible \\
\hline Pouna & $2.00^{\mathrm{ab}}$ & $19252.27 b^{c}$ & $3.85^{\mathrm{ab}}$ & Susceptible \\
\hline TDr 97/00793 & $2.00^{\mathrm{ab}}$ & $19694.14^{\mathrm{ab}}$ & $3.94^{\mathrm{ab}}$ & Susceptible \\
\hline TDr 95/18544 & $2.50^{\mathrm{ab}}$ & $20842.36^{\mathrm{ab}}$ & $4.17^{\mathrm{ab}}$ & Susceptible \\
\hline TDr 99/02674 & $1.50^{\mathrm{bc}}$ & $24021.32^{\mathrm{ab}}$ & $4.80^{\mathrm{a}}$ & Susceptible \\
\hline TDr 97/00940 & $2.00^{\mathrm{ab}}$ & $24904.30^{\mathrm{ab}}$ & $4.98^{\mathrm{a}}$ & Susceptible \\
\hline TDr 96/01817 & $2.00^{\mathrm{ab}}$ & $25434.02^{\mathrm{ab}}$ & $5.09^{\mathrm{a}}$ & Susceptible \\
\hline TDr 89/02665 & $1.75^{\mathrm{b}}$ & $25522.10^{\mathrm{ab}}$ & $5.10^{\mathrm{a}}$ & Susceptible \\
\hline TDr 00/00403 & $2.00^{\mathrm{ab}}$ & $25876.33^{\mathrm{ab}}$ & $5.18^{\mathrm{a}}$ & Susceptible \\
\hline TDr 06-15 & $2.00^{\mathrm{ab}}$ & $30203.05^{\mathrm{ab}}$ & $6.04^{\mathrm{a}}$ & Susceptible \\
\hline TDr 89/02672 & $1.75^{\mathrm{b}}$ & $36915.17^{\mathrm{a}}$ & $7.38^{\mathrm{a}}$ & Susceptible \\
\hline
\end{tabular}

Note. Values are means of three replicates. Means with same letter in the same column are not significantly different at $\mathrm{P}=0.05$ using the Student-Newman-Keuls Test. Reproductive factor $(\mathrm{RF})=\mathrm{Pf} / \mathrm{Pi}$ where $\mathrm{Pf}$ is final nematode population (in soil, roots and tubers) and Pi is initial population. Damage score: $1=0 \%$ damage, $2=$ $1-10 \%$ damage, $3=11-30 \%$ damage, $4=31-60 \%$ damage, $5=61-100 \%$ damage. Host Status Scheme: Resistant $=\mathrm{RF} \leq 1, \mathrm{GI} \leq 2 ;$ Tolerant $=\mathrm{RF} \leq 1, \mathrm{GI} \geq 2 ;$ Susceptible $=\mathrm{RF} \geq 1, \mathrm{GI} \geq 2$. 
Table 3. Nematode population, gall index, reproductive factor and host status of accessions innoculated with Meloidogyne spp. in the second trial

\begin{tabular}{|c|c|c|c|c|}
\hline Accession & Gall-Index & Nematode Count & Reproductive factor & Host status \\
\hline TDr 01/00405 & $2.00^{\mathrm{bc}}$ & $1240.26^{\mathrm{b}}$ & $0.25^{\mathrm{d}}$ & Resistant \\
\hline TDr 08-3-6 & $2.00^{\mathrm{bc}}$ & $1265.16^{\mathrm{b}}$ & $0.25^{\mathrm{d}}$ & Resistant \\
\hline TDr 94/01108 & $2.00^{\mathrm{bc}}$ & $1340.18^{\mathrm{b}}$ & $0.27^{\mathrm{d}}$ & Resistant \\
\hline TDr 98/00205 & $1.00^{\mathrm{c}}$ & $1340.41^{\mathrm{b}}$ & $0.27^{\mathrm{d}}$ & Resistant \\
\hline TDr $03 / 00180$ & $1.00^{\mathrm{c}}$ & $1360.15^{\mathrm{b}}$ & $0.27^{\mathrm{d}}$ & Resistant \\
\hline TDr 96/00582 & $2.00^{\mathrm{bc}}$ & $1365.22^{\mathrm{b}}$ & $0.27^{\mathrm{d}}$ & Resistant \\
\hline TDr $02 / 00515$ & $2.00^{\mathrm{bc}}$ & $6215.15^{\mathrm{b}}$ & $1.24^{\mathrm{d}}$ & Susceptible \\
\hline TDr 97/01715 & $2.00^{\mathrm{bc}}$ & $6225.46^{\mathrm{b}}$ & $1.25^{\mathrm{d}}$ & Susceptible \\
\hline Alumaco & $2.00^{\mathrm{bc}}$ & $6245.04^{\mathrm{b}}$ & $1.25^{\mathrm{d}}$ & Susceptible \\
\hline TDr $89 / 02665$ & $2.00^{\mathrm{bc}}$ & $6265.11^{\mathrm{b}}$ & $1.25^{\mathrm{d}}$ & Susceptible \\
\hline TDr $89 / 02677$ & $3.00^{\mathrm{ab}}$ & $7265.41^{\mathrm{b}}$ & $1.45^{\mathrm{d}}$ & Susceptible \\
\hline TDr 89/02157 & $2.00^{\mathrm{bc}}$ & $7340.25^{\mathrm{b}}$ & $1.46^{\mathrm{d}}$ & Susceptible \\
\hline TDr 96/01817 & $1.25^{\mathrm{c}}$ & $10030.42^{\mathrm{b}}$ & $2.00^{\mathrm{cd}}$ & Susceptible \\
\hline TDr $07 / 00873$ & $2.70^{\mathrm{ab}}$ & $10180.39^{\mathrm{b}}$ & $2.04^{\mathrm{b}}$ & Susceptible \\
\hline TDr 00/00539 & $3.00^{\mathrm{ab}}$ & $10490.87^{\mathrm{b}}$ & $2.10^{\mathrm{d}}$ & Susceptible \\
\hline TDr 06-15 & $2.75^{\mathrm{ab}}$ & $10495.13^{\mathrm{b}}$ & $2.10^{\mathrm{d}}$ & Susceptible \\
\hline TDr $07 / 00168$ & $1.53^{\mathrm{bc}}$ & $16490.21^{\mathrm{ab}}$ & $2.30^{\mathrm{c}}$ & Susceptible \\
\hline TDr 99/02674 & $2.50^{\mathrm{ab}}$ & $11500.12^{\mathrm{b}}$ & $2.30^{\mathrm{d}}$ & Susceptible \\
\hline TDr 99/02789 & $2.50^{\mathrm{ab}}$ & $11510.37^{\mathrm{b}}$ & $2.30^{\mathrm{d}}$ & Susceptible \\
\hline TDr 95/01932 & $2.50^{\mathrm{ab}}$ & $12025.29^{b}$ & $2.41^{\mathrm{d}}$ & Susceptible \\
\hline TDr 00/00403 & $2.50^{\mathrm{ab}}$ & $12120.33^{b}$ & $2.42^{\mathrm{d}}$ & Susceptible \\
\hline TDr $95 / 19177$ & $3.55^{\mathrm{a}}$ & $12475.28^{\mathrm{b}}$ & $2.48^{\mathrm{d}}$ & Susceptible \\
\hline TDr 89/02672 & $2.25^{\mathrm{bc}}$ & $12575.28^{\mathrm{b}}$ & $2.50^{\mathrm{d}}$ & Susceptible \\
\hline TDr 97/00840 & $2.00^{\mathrm{bc}}$ & $12620.08^{\mathrm{b}}$ & $2.52^{\mathrm{d}}$ & Susceptible \\
\hline TDr 03/00196 & $2.00^{\mathrm{bc}}$ & $12650.11^{\mathrm{b}}$ & $2.53^{\mathrm{d}}$ & Susceptible \\
\hline TDr $03 / 00058$ & $2.00^{\mathrm{bc}}$ & $12660.10^{\mathrm{b}}$ & $2.53^{\mathrm{d}}$ & Susceptible \\
\hline TDr 96/00604 & $2.00^{\mathrm{bc}}$ & $12780.32^{\mathrm{b}}$ & $2.55^{\mathrm{d}}$ & Susceptible \\
\hline TDr 97/00793 & $2.50^{\mathrm{ab}}$ & $13115.32^{\mathrm{b}}$ & $2.62^{\mathrm{cd}}$ & Susceptible \\
\hline TDr 95/18544 & $2.50^{\mathrm{ab}}$ & $13400.17^{\mathrm{b}}$ & $2.68^{\mathrm{cd}}$ & Susceptible \\
\hline Ufenyi & $2.00^{\mathrm{bc}}$ & $13545.14^{\mathrm{b}}$ & $2.71^{\mathrm{cd}}$ & Susceptible \\
\hline Amula & $2.00^{\mathrm{bc}}$ & $13675.40^{\mathrm{b}}$ & $2.74^{\mathrm{cd}}$ & Susceptible \\
\hline TDr 00/00362 & $2.70^{\mathrm{ab}}$ & $13910.38^{\mathrm{ab}}$ & $2.78^{\mathrm{cd}}$ & Susceptible \\
\hline TDr 95/19158 & $4.00^{\mathrm{a}}$ & $13995.23^{\mathrm{ab}}$ & $2.80^{\mathrm{cd}}$ & Susceptible \\
\hline TDr 89/02475 & $3.00^{\mathrm{ab}}$ & $14190.01^{\mathrm{ab}}$ & $2.84^{\mathrm{cd}}$ & Susceptible \\
\hline TDr $89 / 00933$ & $2.00^{\mathrm{bc}}$ & $14270.08^{\mathrm{ab}}$ & $2.85^{\mathrm{cd}}$ & Susceptible \\
\hline TDr 99/02562 & $2.75^{\mathrm{ab}}$ & $17390.27^{\mathrm{a}}$ & $3.48^{\mathrm{c}}$ & Susceptible \\
\hline TDr 97/00917 & $2.00^{\mathrm{bc}}$ & $17830.32^{\mathrm{a}}$ & $3.57^{\mathrm{c}}$ & Susceptible \\
\hline TDr 97/00940 & $2.00^{\mathrm{bc}}$ & $18735.18^{\mathrm{a}}$ & $3.75^{\mathrm{c}}$ & Susceptible \\
\hline TDr 99/02607 & $3.75^{\mathrm{a}}$ & $22380.36^{\mathrm{a}}$ & $4.48^{\mathrm{a}}$ & Susceptible \\
\hline
\end{tabular}

Note. Values are means of three replicates. Means with same letter in the same column are not significantly different at $\mathrm{P}=.05$ using the Student-Newman-Keuls Test. Reproductive factor $(\mathrm{RF})=\mathrm{Pf} / \mathrm{Pi}$ where $\mathrm{Pf}$ is final nematode population (in soil, roots and tubers) and Pi is initial population. Damage score: $1=0 \%$ damage, $2=$ $1-10 \%$ damage, $3=11-30 \%$ damage, $4=31-60 \%$ damage, $5=61-100 \%$ damage. Host Status Scheme: Resistant $=\mathrm{RF} \leq 1, \mathrm{GI} \leq 2$; Tolerant $=\mathrm{RF} \leq 1, \mathrm{GI} \geq 2$; Susceptible $=\mathrm{RF} \geq 1, \mathrm{GI} \geq 2$. 

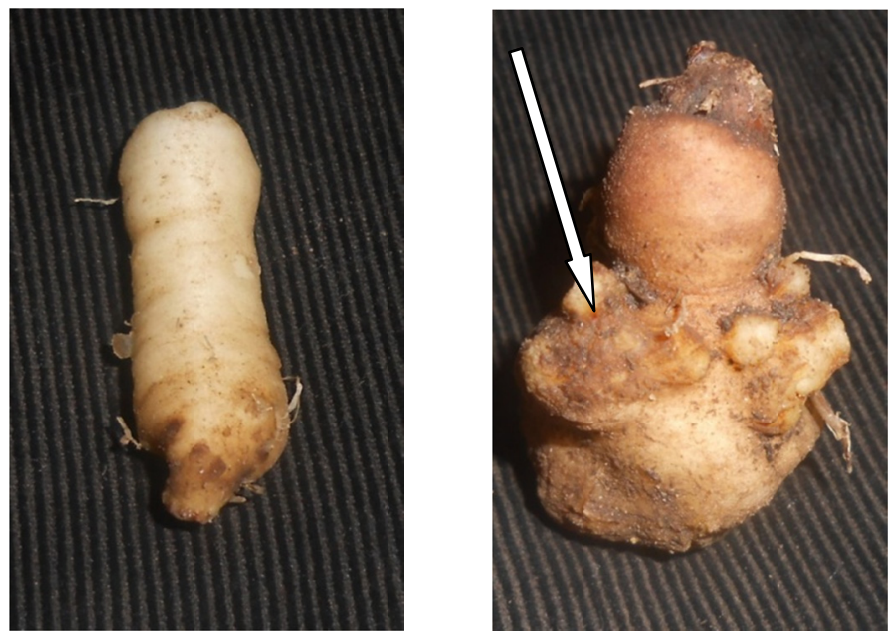

Plate 2. Uninoculated yam tuber (a) and galled and deformed tuber generated from vine cuttings

TDr 08-3-6, TDr 01/00405 and TDr 94/01108 were designated as resistant based on low damage indices and low nematode reproductive factor (Table 3). Cultivar TDr 96/00582 which was susceptible in the first trial was categorized as resistant in the second trial, while TDr 07/00168 which was resistant in the first trial was listed as susceptible in the second trial. All other cultivars were designated as susceptible with TDr 99/02607 having the highest nematode populations and reproductive factor. Yam tubers that were uninoculated with nematodes (control) had smooth appearance and without blemishes (Plate 2) while tubers from inoculated plants were galled and deformed, characteristic of Meloidogyne spp. damage.

\section{Discussion}

The survival rate of vines in this study was about $60 \%$ in the second trial where there were no differences between inoculated and uninoculated plants. Although in the first trial survival rate was lower and significantly so for inoculated plants. This was due to mite infestation in the screen house during that season, where many of the plants lost leaves. The loss of leaves however, did not affect the tuberization of plants as tubers were collected from most of the planted holes. Otoo et al. (2016) reported an average of 75\% establishment rate of vines cuttings at two weeks after planting. Although Claudius-Cole et al. (in press) observed a survival rate ranging between $67-78 \%$ at four weeks after planting and nematode- inoculated vines with a survival rate of $62-66 \%$.

The missing cultivars in the trials further proves how availability of tubers may contribute to challenges of screening procedure for yams. The vine system yielded mini-tubers, even in plants that did not show evidence of increased vine length or number of leaves. The tubers varied in size and length across varieties and treatments. While, most of the inoculated plants produced tubers lower in weight than the control, some cultivars that were inoculated weighed significantly more than the control tubers. This is not unexpected because infestation by Meloidogyne spp. normally induces the production of growth hormones that cause excessive cell growth and proliferation that is visible as galls on the surface of tubers (Williamson \& Hussey, 1996; Kwoseh, 2000). Therefore when there are many galls on such tubers, their weight could be more than those not infected with the nematode. Nwauzor and Fawole (1981), Kwoseh (2000), and Moens, Perry, and Starr (2009) showed in their studies that, Meloidogyne spp. on yam adversely affects their shape and appearance thereby reducing their marketability and value. They also demonstrated that there may be no weight reduction in infected tubers at harvest.

Dioscorea rotundata is the most widely grown and consumed yam species in West Africa although the water yam may yield better. Results from this study show that $D$. rotundata is highly prone to damage by the root-knot nematode and this can lead to further deterioration in storage causing severe storage losses. There were clear differences between the Meloidogyne-inoculated and control plants in terms of tuber damage scoring and recovered nematode populations, indicating that the method is effective for showing nematode infection. Of the 40 cultivars screened in this study, five, TDr 98/00205, TDr 03/00180, TDr 08-3-6, TDr 01/00405 and TDr 94/01108 consistently showed resistant reaction to Meloidogyne spp. in both trials while the other accessions mostly showed susceptible reaction to the nematode. Cultivars of $D$. rotundata were reported to be more 
susceptible to the root-knot nematode than D. alata, D. cayenensis and D. bulbifera (Ogbuji, 1978). The resistant reaction of the five cultivars may be due to the presence of resistant genes which can be utilized by breeders along with desirable agronomic characteristics to develop varieties with better performance against this constraint. Development of $D$. rotundata varieties with nematode resistance is valuable in managing root-knot nematode populations and limiting their damage on succeeding crops in intensive cropping systems with limited use of chemicals. One cultivar TDr 99/02562 was designated as tolerant in the first trial but did not maintain this status in the second trial. The tuber weight for this particular variety was the highest in the first trail. Probably, early tuberization of the variety may have resulted in avoidance of penetration of many juveniles. Conversely, TDr $07 / 00168$ which was resistant only in the first trial may have been due to the very small tuber size, implying that there may have been a delay in tuberization and probably nematode infestation compared to the second trial.

The use of vine cuttings as planting material for the study was found to be an effective method for evaluating the reaction of yam germplasm to the root-knot nematode. The use of hanging bags as a substitute of conventional pots, increased the efficiency of the space in the screenhouse because the bags were organized in layers. One bag also represented a replicate containing 10 plants, thus reducing the variability of the results. It also has great potential for research (Behera et al., 2009) as the technique offers a solution to the various challenges in using the conventional method of planting mini-setts in pots or fields. These challenges include uneven sprouting of tubers, sufficient replication due to insufficient tubers, nematode inoculum requirement per plant, and space requirements for large number of accessions. The method also eliminates the waiting time for dormancy breaking in tubers compared to if tubers or setts are used. When tubers of required varieties are few or scarce, the use of vine cuttings can serve to provide enough planting material for experimental purposes, especially for screening for disease resistance while obtaining similar results as for conventional screening methods.

\section{References}

Adegbite, A. A., \& Agbaje, G.O. (2007). Efficacy of carbofuran in control of root-knot nematode (Meloidogyne incognita race 2) in hybrid yam varieties in southwestern Nigeria. Electronic Journal of Environment, Agriculture and Food Chemistry, 6, 2083-2094.

Atu, U. G., Odurukwe, S. O., \& Ogbuji, R. O. (1983). Root-knot nematode damage to Dioscorea rotundata. Plant Disease, 67, 814-815. https://doi.org/10.1094/PD-67-814

Behera, K. K., Sahoo, S., Maharana, T., \& Pani, D. (2009). Response of vine cuttings to rooting in different months in three Dioscorea species. Nature and Science, 7(2), 48-51.

Canto-Saenz, M. (1983). The nature of resistance to Meloidogyne incognita (Kofoid \& White) Chitwood. In C. C. Carter (Ed.), Proceeding, third research \& planning conference on root-knot nematodes, Meloidogyne spp. (p. 233). International Meloidogyne Project, Lima, Peru.

Correl, D. S., Schubert, B. G., Gentry, H. S., \& Hawley, W. D. (1955). The search for plant precursors of cortisone. Economic Botany, 9, 307-375. https://doi.org/10.1007/BF02985309

Coyne, D. L., \& Ross, J. L. (2014). Protocol for Nematode Resistance Screening: Root knot Nematodes, Meloidogyne spp. (p. 27). International Institute of Tropical Agriculture (IITA), Ibadan, Nigeria.

Coyne, D. L., Nicol, J. M., \& Claudius-Cole, B. (2007). Practical Plant Nematology: A Field and Laboratory Guide (p. 82). SP-IPM, International Institute of Tropical Agriculture (IITA), Cotonou, Benin.

Egesei, C. N., Onyeka, T. J., \& Asiedu, R. (2007). Severity of anthracnose and virus diseases of water yam (Dioscorea alata L.) in Nigeria I: Effects of yam genotype and date of planting. Crop Protection, 26, 1259-1265. https://doi.org/10.1016/j.cropro.2006.10.025

FAO (Food and Agricultural Organisation of the United Nations). (2012). Production Yearbook FAO Statistics 2011. Rome, Italy.

Hashem, M., \& Abo-Elyousr, K. A. (2011). Management of the root-knot nematode Meloidogyne incognita on tomato with combinations of different biocontrol organisms. Crop Protection, 30(3), 285-292. https://doi.org/10.1016/j.cropro.2010.12.009

Hussey, R. S., \& Barker, K. R. (1973). A comparison of methods of collecting inocula of Meloidogyne spp., including a new technique. Plant Disease Reporter, 57, 1025-1028.

Ile, E. I., Craufurd, P. Q., Battey, N. H., \& Asiedu, R. (2006). Phases of Dormancy in Yam Tubers (Dioscorea rotundata). Annals of Botany, 97(4), 497-504. http://doi.org/10.1093/aob/mc1002 
Kiewnick, S., \& Sikora, R. A. (2006). Biological control of the root-knot nematode Meloidogyne incognita by Paecilomyces lilacinus strain 251. Biological Control, 38, 179-187. https://doi.org/10.1016/j.biocontrol. 2005.12.006

Kwoseh, C. K. (2000). Identification of resistance to major nematode pests of yams (Dioscorea spp.) in West Africa (p. 196, PhD Thesis, The University of Reading, UK).

Moens, M., Perry, R. N., \& Starr, J. L. (2009). Meloidogyne species-A diverse group of novel and important plant parasites. In R. N Perry, M. Moens, \& J. L. Starr (Eds.), Root-knot nematodes (pp. 1-17). CAB International, Wallingford, UK. https://doi.org/10.1079/9781845934927.0001

Nwauzor, E. C., \& Fawole, B. (1981). Root-knot nematodes on yams in eastern Nigeria. Proceedings of the 3rd Research Planning Conference on Root-knot Nematodes, Meloidogyne spp. Region $1 V$ and V (Vol. 16, pp. 1-167). Ibadan, Nigeria.

Nyczepir, A. P., \& Thomas, S. H. (2009). Current and future management in intensive crop production systems. In R. N. Perry, M. Moeans, \& J. L. Starr (Eds.), Root-knot Nematodes (pp. 412-435). CAB International, Wallington, UK. https://doi.org/10.1079/9781845934927.0412

Odu, B. O., Asiedu, R., Hughes, J. d'A., Shoyinka, S., \& Oladiran, A. O. (2004). Identification of resistance to Yam mosaic virus (YMV), genus Potyvirus in white yam (Dioscorea rotundata). Field Crops Research, 89, 97-105. https://doi.org/10.1016/j.fcr.2004.01.009

Ogbuji, R. O. (1978). Damage caused by Meloidogyne incognita association with soil borne fungi in ten taro cultivars in Nigeria. Ghana Journal of Agric. Science, 11, 161-164.

Otoo, E., Anyakanmi, T. G., Kikuno, H., \& Asiedu, R. (2016). In Vivo Yam (Dioscorea spp.) Vine Multiplication Technique: The Plausible Solution to Seed Yam Generation Menace. Journal of Agricultural Science, 8(2), 88-97. https://doi.org/10.5539/jas.v8n2p88

Sahebani, N., \& Hadavi, N. (2008). Biological control of the root knot nematode Meloidogyne javanica by Trichoderma harzianum. Soil Biology and Biochemistry, 40, 2016-2020. https://doi.org/10.1016/j.soilbio. 2008.03.011

Shiwachi, H., Kikuno, H., \& Asiedu, R. (2005). Micro tuber production using yam (D. rotundata) vines. Tropical Science, 45(4), 163-169. https://doi.org/10.1002/ts.22

Vander Zaag, P., \& Fox, R. L. (1981). Field production of yams (Dioscorea alata) from stem cuttings. Tropical Agriculture, 58, 143-145.

Williamson, V. M., \& Hussey, R. S. (1996). Nematode pathogenesis and resistance in plants. Plant Cell, 8, 1735-1745. https://doi.org/10.1105/tpc.8.10.1735

\section{Copyrights}

Copyright for this article is retained by the author (s), with first publication rights granted to the journal.

This is an open-access article distributed under the terms and conditions of the Creative Commons Attribution license (http://creativecommons.org/licenses/by/4.0/). 